\title{
Improved photometric redshifts with colour-constrained galaxy templates for future wide-area surveys
}

\author{
Bomee Lee ${ }^{\circledR \star}$ and Ranga-Ram Chary \\ MS314-6, Infrared Processing and Analysis Center, California Institute of Technology, Pasadena, CA 91125, USA
}

Accepted 2020 July 8. Received 2020 June 20; in original form 2019 October 22

\begin{abstract}
Cosmology and galaxy evolution studies with LSST, Euclid, and Roman, will require accurate redshifts for the detected galaxies. In this study, we present improved photometric redshift estimates for galaxies using a template library that populates threecolour space and is constrained by HST/CANDELS photometry. For the training sample, we use a sample of galaxies having photometric redshifts that allows us to train on a large, unbiased galaxy sample having deep, unconfused photometry at opticalto-mid infrared wavelengths. Galaxies in the training sample are assigned to cubes in 3D colour space, $V-H, I-J$, and $z-H$. We then derive the best-fitting spectral energy distributions of the training sample at the fixed CANDELS median photometric redshifts to construct the new template library for each individual colour cube (i.e. colour-cube-based template library). We derive photometric redshifts (photo- $z$ ) of our target galaxies using our new colour-cube-based template library and with photometry in only a limited set of bands, as expected for the aforementioned surveys. As a result, our method yields $\sigma_{\mathrm{NMAD}}$ of 0.026 and an outlier fraction of 6 per cent using only photometry in the LSST and Euclid/Roman bands. This is an improvement of $\sim 10$ per cent on $\sigma_{\text {NMAD }}$ and a reduction in outlier fraction of $\sim 13$ per cent compared to other techniques. In particular, we improve the photo- $z$ precision by about 30 per cent at $2<z<3$. We also assess photo- $z$ improvements by including $K$ or mid-infrared bands to the ugrizYJH photometry. Our colour-cube-based template library is a powerful tool to constrain photometric redshifts for future large surveys.
\end{abstract}

Key words: methods: statistical-techniques: photometric-galaxies: photometry.

\section{INTRODUCTION}

Upcoming deep, wide-area surveys with Euclid, Nancy Grace Roman Space Telescope (Roman), and the Legacy Survey of Space and Time (LSST) of the Vera C. Rubin Observatory will cover several thousands of square degrees of sky and observe billions of sources. Robust redshift estimates of such large numbers of galaxies are required both for galaxy and cluster evolution studies and $3 \mathrm{D}$ weak lensing analysis. Ideally, spectroscopic redshifts yield the most accurate distances. However, spectroscopic surveys are biased towards strong emission line galaxies, are severely flux-limited, and biased against fainter galaxies. Obtaining spectroscopic redshifts of billions of objects is unfeasible, especially for faint galaxies that constitute the dominant fraction of the sample. Therefore, these future large surveys have to rely on photometric redshifts. This allows us to measure distances to most galaxies with detections in multiple bands to very faint flux density levels with good statistical accuracy. More accurate and precise photo- $z$ estimation is a challenging task, especially for the cosmological weak gravitational lensing measurements that have a stringent redshift bias requirement $\left(\Delta_{z}<0.002\right.$; Laureijs et al. 2011).

Photometric redshifts are determined by developing and applying the mapping between redshift, observed flux densities and colours, using either template fitting (e.g. LEPHARE, EAZY, etc.) or machinelearning techniques. In recent years, many machine-learning ap-

^E-mail: bomee@ipac.caltech.edu proaches have been developed (Collister \& Lahav 2004; Carrasco Kind \& Brunner 2013, 2014; Rau et al. 2015; Hoyle 2016) and it has become popular because it is fast and powerful without the large systematic uncertainties associated with modelling dust and emission-line contributions (Speagle \& Eisenstein 2017a). While machine-learning techniques are effective within their training sets (Sánchez et al. 2014; Hoyle et al. 2015; Masters et al. 2015; Speagle \& Eisenstein 2017b; Bilicki et al. 2018; Fotopoulou \& Paltani 2018), the mapping between various colours (or flux densities) and redshift is not based on pre-existing physical knowledge, but is newly obtained every time, using a training sample with photometry and known redshifts. Therefore, machine-learning heavily depends on a training sample with spectroscopic redshifts, which are limited to brighter sources. Besides, Masters et al. (2015) showed spectroscopic redshifts have thus far, missed significant regions of colour space that has motivated colour-space targeted redshift surveys such C3R2 (Masters et al. 2017).

In this study, we provide a new method to improve photometric redshift estimates using the template-fitting approach. While templatefitting approaches are fundamentally limited by the accuracy of existing templates and quality of the photometry, we implement a hybrid approach combining the methodology of both template fitting and empirical information obtained from a training sample. This relies on correlations between redshifts and observables such as colours, magnitude, and galaxy morphology. It is well known that photometric tracers of strong features such as the Lyman break at $912 \AA$, Balmer break at $4000 \AA$, and the $1.6 \mu \mathrm{m}$ bump provide redshift 
constraints (Sawicki 2002; Salvato, Ilbert \& Hoyle 2019). It is also well known that high-redshift galaxies have fainter magnitudes and smaller sizes (e.g. van der Wel et al. 2014, and references therein) than the local Universe. We aim to incorporate such information about the evolution of galaxy properties in multidimensional parameter space obtained from a training sample, to the template-fitting approach. Instead of the usual training set with spectroscopic redshifts, our training set consists of galaxies with known photometric redshifts derived as the average of multiple fitting techniques. The accuracy of photometric redshifts depends on the quality of photometry (high spatial resolution and high signal to noise) and wide spectral coverage with many photometric bands sampling the spectral energy distribution (SED; Brammer, van Dokkum \& Coppi 2008; Dahlen et al. 2010). For our purpose, the HST/Cosmic Assembly Nearinfrared Extragalactic Legacy Survey (CANDELS; Grogin et al. 2011; Koekemoer A., Faber S. \& Ferguson H. C. et 2011) is the optimal data set to train galaxy SED templates, as it has excellent spectral coverage from the X-rays to far-infrared with exceptional depth and spatial resolution. Fotopoulou \& Paltani (2018) presented a similar hybrid approach identifying the optimal photometric redshift model library for each source class before performing SED fitting, but they use a machine-learning algorithm trained with spectroscopic redshifts to classify the model templates. They found that their method is advantageous for reducing catastrophic outliers for all types of galaxies and deriving photometric redshifts for AGN and QSOs, while the photometric redshift scatter $(\sigma=0.033-0.038)$ is slightly worse compared to the pure template fitting and machinelearning methods.

Here, we do not aim to invent new template models or a fitting code. We leverage HST/CANDELS photometry to constrain the range of templates and apply those to derive improved photometric redshifts for future wide-area surveys. The structure of this paper as follows. CANDELS data is introduced in Section 2 together with selections of the training and target sample. We describe the method used to construct the new template library that populates $3 \mathrm{D}$ colour space, and derive the photometric redshifts using our new template library in Section 3. In Section 4, we apply our method to the photometry expected from the LSST and Euclid-deep/Roman fields and validate the photometric redshift performance. We conclude in Section 5.

All magnitudes are expressed in the $\mathrm{AB}$ system unless stated otherwise. We use a standard $\Lambda$ CDM cosmology with $H_{0}=70 \mathrm{~km} \mathrm{~s}^{-1} \mathrm{Mpc}^{-1}, \Omega_{\mathrm{M}}=0.3$, and $\Omega_{\Lambda}=0.7$, which is broadly consistent with the recent results from Planck.

\section{CANDELS DATA}

We use HST/CANDELS (Grogin et al. 2011; Koekemoer et al. 2011) observations that span five well-studied extragalactic fields: the Great Observatories Origins Deep Survey (GOODS) Northern and Southern fields (GOODS-North and GOODS-South), the UKIRT InfraRed Deep Sky Surveys Ultra Deep Field (UDS), the Extended Groth Strip (EGS) field, the Cosmic Evolution Survey (COSMOS) field. These fields together map out about $900 \mathrm{arcmin}^{2}$ of sky achieving $5 \sigma$ point source limiting depth of $\sim 27.3 \mathrm{AB}$ mag (wide) and $\sim 28.0 \mathrm{AB}$ mag (deep) in the $\mathrm{H}$ (F160W) band. CANDELS multiwavelength photometry catalogues span from the $U$ band through $8 \mu \mathrm{m}$ and are publicly available in each field (GOODS-S, Guo et al. 2013; GOODS-N, Barro et al. 2019; UDS, Galametz et al. 2013; EGS, Stefanon et al. (2017; COSMOS; Nayyeri et al. 2017). Each field of CANDELS has been observed with many different telescopes and instruments in many different wavelengths. The multiple bands we use in this study for each field are listed next. (i) GOODS-S (GS): A total of 17 bands with U(Blanco/Mosaic II or VLT/VIMOS), F435W, F606W, F775W, F814W, F850LP from HST/ACS, F098M, F105W, F125W, F160W from HST/WFC3, Ks (VLT/ ISAAC and HAWK-I), 3.6, 4.5, 5.8, $8.0 \mu \mathrm{m}$ from Spitzer/IRAC.

(ii) GOODS-N (GN): A total of 16 bands with U(KPNO/Mosaic), F435W, F606W, F775W, F814W, F850LP from HST/ACS, F105W, F125W, F140W, F160W from HST/WFC3, Ks (Subaru/MOIRCS), $\mathrm{K}$ (CFHT/MegaCam), 3.6, 4.5, 5.8, $8.0 \mu \mathrm{m}$ from Spitzer/IRAC.

(iii) UDS: A total of 17 bands with U (CFHT/MegaCam), $B, V$, $R, i, z$ from Subaru/Suprime-Cam, F606W, F814W from HST/ACS, F125W, F160W from HST/WFC3, Y and Ks from VLT/HAWK-I, K from UKIRT/WFCAM, 3.6, 4.5, 5.8, $8.0 \mu \mathrm{m}$ from Spitzer/IRAC.

(iv) EGS: A total of 20 bands with $u, g, r, i, z$, from CFHT/ MegaCam, F606w, F814W from HST/ACS, F125W, F140W, F160W from $H S T / W F C 3$, J1, J2, J3, H1, H2 from Mayall/NEWFIRM, Ks from CFHT/WIRCAM, 3.6, 4.5, 5.8, $8.0 \mu \mathrm{m}$ from Spitzer/IRAC.

(v) COSMOS: A total of 33 bands with $u, g, r, i, z$ from CFHT/MegaPrime, B from Subaru/Suprime-Cam, F606W, F814W from HST/ACS, F125W, F160W from HST/WFC3, Y and Ks from VISTA/VIRCAM, J1, J2, J3, H1, H2 from Mayall/NEWFIRM, 3.6, 4.5, 5.8, $8.0 \mu \mathrm{m}$ from Spitzer/IRAC, and 12 intermediate bands in optical (IA484-IB827) from Subaru/Suprime-Cam.

We correct the photometry in the catalogues for Galactic extinction using the value given by the IRSA Galactic dust reddening and extinction calculator. ${ }^{1}$ Extinction values are calculated at the centre of each field based on Schlafly \& Finkbeiner (2011) and are provided for a small set of filters. We then interpolate between the values to determine the extinction at the central wavelength of each filter in our data set. The median $g$-band extinction in the five fields is 0.040 , 0.026, 0.061, 0.074, and $0.027 \mathrm{mag}$ for GN, GS, COSMOS, UDS, and EGS, respectively. We note that applying the Galactic extinction correction slightly reduces the photometric redshift scatter by about 2-3 per cent within the CANDELS fields.

It is worth noting that at depths beyond $\sim 25 \mathrm{AB}$ mag in the optical, source confusion in seeing-limited imaging biases photometry (See Fig. 1; e.g. Joint Survey Processing report 2019). The Hubblequality spatial resolution of the deep optical data in the catalogues, combined with the priors on morphology and galaxy shapes applied for catalogueing alleviates much of the confusion (Merlin et al. 2016). Thus, using the CANDELS catalogues, we have the highest quality photometry available to build-up the SED of the training sample of galaxies.

\subsection{Training sample}

The galaxies in the training sample are required to have signal-tonoise ratio $(\mathrm{S} / \mathrm{N})>3$ in $\mathrm{U}, \mathrm{B}(\mathrm{F} 435 \mathrm{~W}), \mathrm{F} 606 \mathrm{~W}, \mathrm{~F} 814 \mathrm{~W}, \mathrm{~F} 125 \mathrm{~W}$, F160W, K (or Ks), and $3.6 \mu \mathrm{m}$ IRAC 1 channel to avoid having photometric uncertainties dominate redshift estimates. We then exclude suspicious sources and stars based on flags provided from the CANDELS photometry catalogue;

(i) Suspicious sources: SExtractor PhotFlag $=0$

(ii) Stars: CLASS_STAR from SExtractor $\geq 0.98$

SExtractor PhotFlag is used to designate suspicious sources that fall in regions of the image that are contaminated by stellar spikes or haloes or edge effects (Guo et al. 2013). Using PhotFlag $=0$, we are

\footnotetext{
${ }^{1}$ https://irsa.ipac.caltech.edu/applications/DUST/
} 


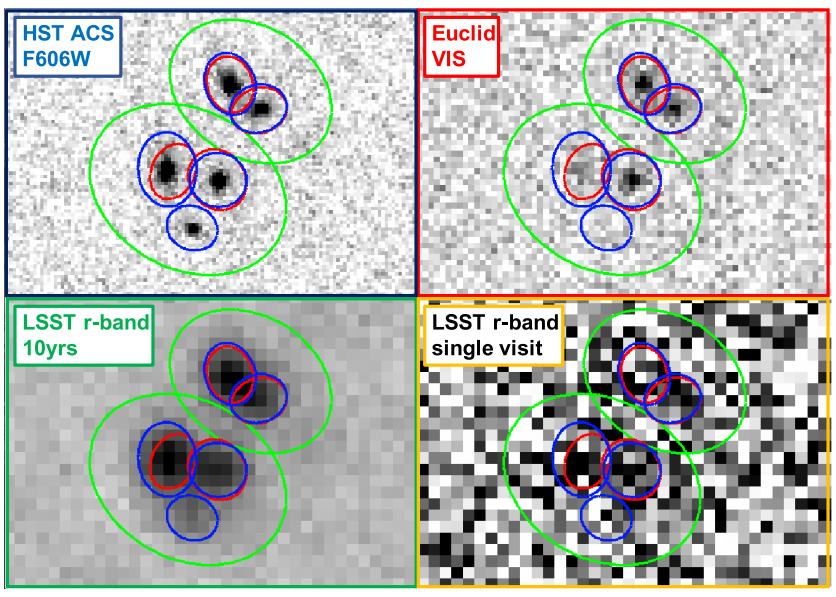

Figure 1. Source confusion in an optical band (centred at $\sim 6000 \AA$ ), along with the isophotes derived from photometry on each of the images. The green isophotes are derived from ground-based resolution $r$-band data of $27.7 \mathrm{AB}$ mag (e.g. LSST), the red isophotes are from the Euclid-quality optical data, while the blue isophotes are from the HST/ACS data. The sources are barely detected in the LSST single epoch data that have a depth of $24.7 \mathrm{AB}$ mag. In the absence of the deeper, space-resolution data, source confusion would result in both erroneous shape and photometry estimates in ground-based data and also affect catalogue matching.

able to exclude detections of star spikes, haloes, and bright stars, as well as excluding sources that are either artifacts or falling at the edge of the image. We also exclude AGN-dominated sources identified from X-ray, IR, and/or radio detections (using the CANDELS catalogue AGN_Flag) that are about 1 per cent of the sample. Since we do not have AGN templates to constrain their redshifts correctly, those sources usually result in higher uncertainties in the photometric redshifts compared to more normal galaxies (Salvato et al. 2009).

To build as large a training set as possible, which is not biased towards strong emission line sources, we consider only galaxies having photometric redshifts, i.e. we exclude all objects with spectroscopic redshifts from the training sample. For that, we use the CANDELS photometric redshifts (photo- $z$ ), which is the median redshift from 11 different photometric redshift codes, each using different template SEDs, and priors. Dahlen et al. (2013) showed that the median redshift results in the best estimates among different photo- $z$ codes. This leaves us with a total of 39391 galaxies as the training sample from all five CANDELS fields.

Fig. 2 shows comparisons between the spectroscopic redshifts and CANDELS median photo- $z$ in five fields with different colours. Spectroscopic redshifts are provided by CANDELS and are selected to have a spec-z quality flag $=1$ which means the spectroscopic data is secure. In addition to 381 spec-zs obtained from the CANDELS COSMOS catalogue, we have collected 972 publicly available speczs in the CANDELS-COSMOS field from zCOSMOS (Lilly et al. 2007), LEGA-C (van der Wel et al. 2016), VUDS (Tasca et al. 2017), COSMOS DEIMOS (Hasinger et al. 2018), and C3R2 (Masters et al. 2019) surveys. In total, we obtain 5539 galaxies in five CANDELS fields after excluding suspicious sources, stars, and AGN candidates using the same flags mentioned in Section 2.1. Including all five fields, the CANDELS median photo- $z$ has yielded a photo- $z$ precision of $\sigma_{\mathrm{NMAD}}=0.019$ and a catastrophic outlier of 5.7 per cent at 0 $<z<6$ (see the definitions of statistics in equation 1). $\sigma_{\text {NMAD }}$ of GOODS-N are very small with 0.005 because of an inclusion of medium-width optical bands (Barro et al. 2019). It is worth noting that the photometric redshift accuracy reported here may not

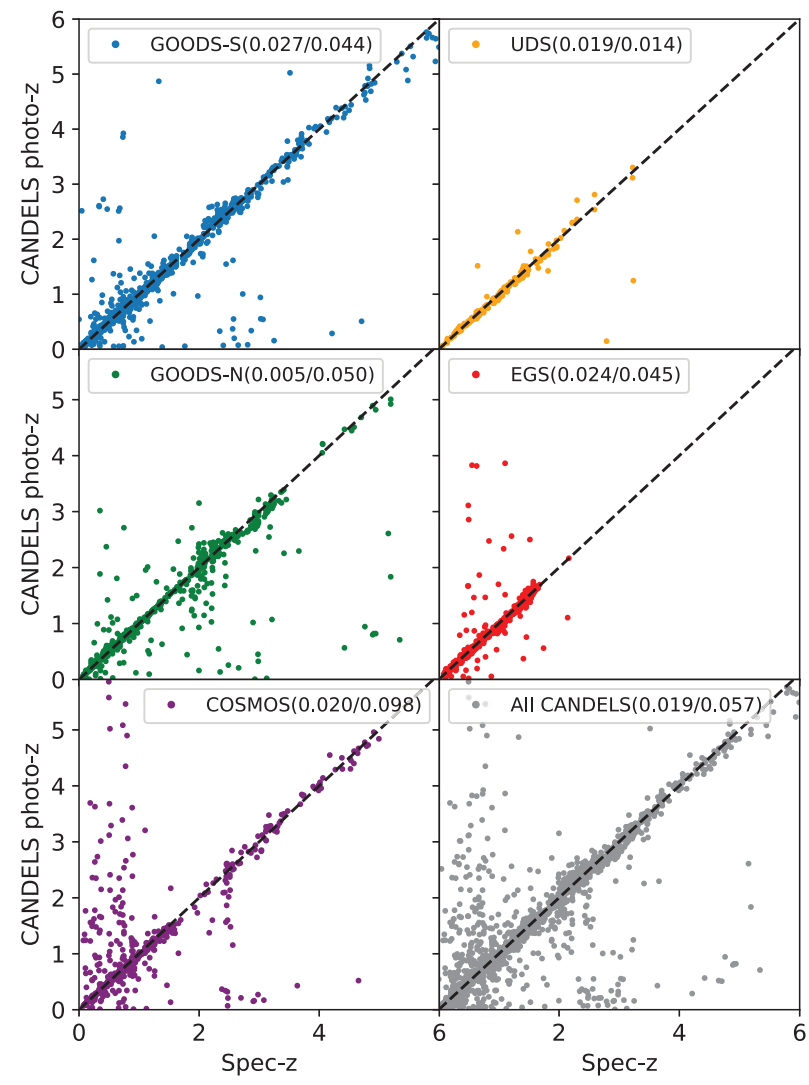

Figure 2. CANDELS median photometric redshifts (photo- $z$ ) versus spectroscopic redshifts (spec-z) in five CANDELS fields. Galaxies are colour-coded by fields; GOODS-S (blue), GOODS-N (green), UDS (orange), EGS (red), COSMOS (purple), and All fields (grey). The numbers in parenthesis are $\sigma_{\text {NMAD }}$ and outlier fraction of each field. There is a relatively low fraction of high redshift galaxies beyond $z_{\text {spec }}=2$, which is about 12 percent the sample with spec-z, and most of them (80 per cent) are in the GOODS-S and $-\mathrm{N}$ fields.

represent galaxies in the entire training sample because the photo$z$ precision and outlier fractions are calculated using a subsample of galaxies with spectroscopic redshifts that are brighter in most case and at lower redshift compared to the full galaxy sample. The accuracy of photometric redshifts are expected to degrade with fainter magnitudes (Ilbert et al. 2009; Dahlen et al. 2013).

There are pros and cons of using a photo-z-selected galaxy sample for developing the training set. Spectroscopically confirmed galaxies tend to be fewer in number, and biased towards bright, emission line galaxies. This can be problematic, especially, for future large photometric surveys that will image billions of galaxies including a large fraction of faint galaxies. Photo- $z$ selected galaxies are an order of magnitude larger in number density, have less redshift accuracy but are relatively unbiased towards galaxies of a particular type. This alleviates the impact of spectroscopic misclassification, which tends to rely on single line redshifts. However, we are not immune from any systematic uncertainties inherent in CANDELS median photo-zs that introduce an additional scatter to our photo- $z$ estimates.

In Fig. 3, we show the distribution of CANDELS median photo$z \mathrm{~s}$ of galaxies in the training sample. In the top panel, the training sample in different fields have photo- $z<3.6$ because we require $3 \sigma$ detection in $U$ band. The red end of the $U$-band filter in CANDELS 

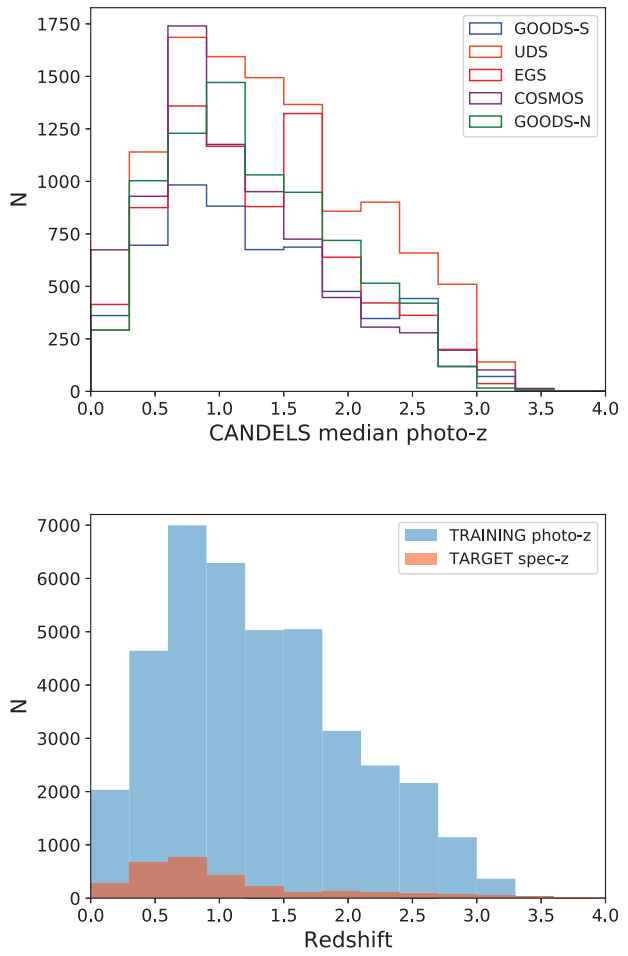

Figure 3. Top: Photo- $z$ distributions of the training sample in five fields, GOODS-S (blue), GOODS-N (green), UDS (orange), EGS (red), COSMOS (purple). Bin size of the histogram is $\Delta z=0.3$. The constraint of $\mathrm{S} / \mathrm{N}>3$ in the $U$ band, restricts galaxies in the training sample to $z<3.6$. Note that the red end of CTIO $U$ band in GOODS-S extends to $4120 \AA$;. Bottom: The photometric redshift distribution of the training sample with a blue histogram and the spectroscopic redshift distribution of the target sample with an orange histogram. Target sample is chosen with galaxies having spectroscopic redshifts at $0<z<6$ in the GOODS-S and $-\mathrm{N}$ fields and having ugrizYJH filters close to LSST-10 yr + Euclid-deep/Roman-quality photometry.

extends to $\sim 4120 \AA$; ; corresponding to the redshifted Lyman-break at $z \sim 3.6$.

\subsection{Target sample}

Future large extragalactic surveys will observe several billions of galaxies and provide multiwavelength catalogues consisting of optical (ugrizy from LSST) and near-infrared (YJH from Euclid ${ }^{2}$, with the addition of F184 from Roman) photometry (See Table 1). The precision of photometric redshifts derived using only these bands will be limited, since it will be challenging for ancillary data sets (e.g. narrow bands) to achieve the unprecedented sensitivities and areal coverage of these surveys. Since our primary goal is to assess the quality of redshifts that can be derived from these surveys, we select 1696 galaxies in the GOODS-S and 1355 galaxies in the GOODS-N fields with spectroscopic redshifts at $0<z<6$ as our target sample as shown in the bottom plot of Fig. 3. We only use photometry in eight filters, including $U$ from the ground-based telescopes, F435W, F606W, F814W, F850p from HST/ACS, and F105W, F125W, F160W from $H S T /$ WFC3, to derive the photo-zs in Section 4.1. We also assess the quality of photo-zs of the target sample with an addition of the IRAC 1 channel in Section 4.2.

${ }^{2}$ Euclid will also image in a broad optical band (VIS) from 550 to $900 \mathrm{~nm}$.
The photometry used here can be considered as a case study for LSST-10 yr+Euclid-deep/Roman-quality photometry albeit they are about a magnitude deeper, except $U$ band as shown in Table 1 . We note that the spatial resolution of the data used here is far superior to that arising from LSST ( 0.1 arcsec versus 0.7 arcsec FWHM), minimizing the impact of source confusion on photometric bias and thereby redshift errors.

In future work, we will apply the constraints from our training set to a completely independent spectroscopic redshift sample, such as that derived from the C3R2 survey (Masters et al. 2017); however, those fields do not have consistently photometered data and deep near-infrared imaging such as that available in the CANDELS field.

\section{METHOD: RECONSTRUCTING GALAXY TEMPLATES IN COLOUR SPACE}

Fig. 4 illustrates four observed colours, $V-H, I-J, H-$ IRAC1, and $z-H$, as a function of galaxy redshift (the grey points), shown along with the median colours for the training (blue) and target (orange) samples. IRAC1 corresponds to the Spitzer/IRAC $3.6 \mu \mathrm{m}$ band. Most of the galaxies in the target sample have similar colours as the training sample, except one galaxy outside the $V-H$ range and three galaxies out of $H$-IRAC1 range. Clearly, there is a smooth and continuous redshift evolution through colour space, showing how multicolour measurements can constrain redshift estimates. Each colour varies with redshift in a different way, reaching a maximum at different redshifts. For example, the peak of the $I-J$ colour is about $z=1.6$ when the Balmer break ( $4000 \AA$;) falls between the $I$ and $J$ bands. For the same reason, $V-H$ peaks at $z=1.1$, and $z-H$ peaks at $z=1.9$. $H-$ IRAC 1 also shows a strong evolution with redshift, especially over $z<1$, due to the $1.6 \mu \mathrm{m}$ bump in galaxy SEDs arising from $\mathrm{H}^{-}$opacity (Sawicki 2002). However, single colours are degenerate in redshift as one can see from the scatter in the greyscales. Stellar population age and dust extinction are degenerate in most optical/NIR colour space such that dusty starburst galaxies can be difficult to distinguish from old, passive galaxies. To constrain redshifts, there only needs to be a distinct spectral feature in the SED of the galaxy. For most such galaxies, it tends to be the $1.6 \mu \mathrm{m}$ bump, so we do not think the age-extinction degeneracy introduces a significant limitation to our redshift estimation. We therefore use three colour information to minimize degeneracies and use the multiwavelength information to apply priors on galaxy SEDs.

\subsection{Cubes in the 3D colour space}

In this study, we settled on using the following three colours after experimenting with various combinations of colour space:

$$
\begin{aligned}
(V-H) & =[-1.53,5.07],(I-J)=[-2.1,3.5], \\
(z-H) & =[-3.1,6.9],
\end{aligned}
$$

where the colour ranges are defined based on CANDELS photometry of the training sample.

Although the relationship between colour and redshift associated with photometric signatures is crucial, we find that the choice of colours strongly relies on the quality of photometry as well. For instance, even though $H-$ IRAC1 is useful to constrain redshifts at $z<1$, we find that $z-H$ improves photo- $z$ estimates better than $H$ -IRAC1 because the $3.6 \mu \mathrm{m}$ imaging is shallower than the $z$ band in our data set. In the template models, the band is also contaminated by $3.3 \mu \mathrm{m}$ emission from polycyclic aromatic hydrocarbons and hot 
Table 1. Comparison between HST/CANDELS and Future surveys with LSST, Euclid, and Roman.

\begin{tabular}{lccc}
\hline Project & Wavelength range & $5 \sigma$ sensitivity $(\mathrm{AB} \mathrm{mag})$ & Spatial resolution $(\operatorname{arcsec})$ \\
\hline HST/CANDELS & VI(ACS) YJH (WFC3) & $>27.4$ & $0.1(\mathrm{VI}) / 0.18(\mathrm{YJH})$ \\
LSST $^{a}$ & ugrizy & $<24.7($ single $) /<27.7(10 \mathrm{yr})$ & 0.7 \\
EuclidNIR $^{b}$ & YJH & $24($ wide $) / 26($ deep $)$ & 0.3 \\
Roman $^{c}$ & $z$ YJH+F184 & $<26.7$ & 0.18 \\
\hline
\end{tabular}

${ }^{a}$ https://docushare.1sst.org/docushare/dsweb/Get/LPM-17

${ }^{b}$ Euclid red book (Laureijs et al. 2011).

${ }^{c}$ WFIRST AFTA 2015 Report (Spergel et al. 2015).
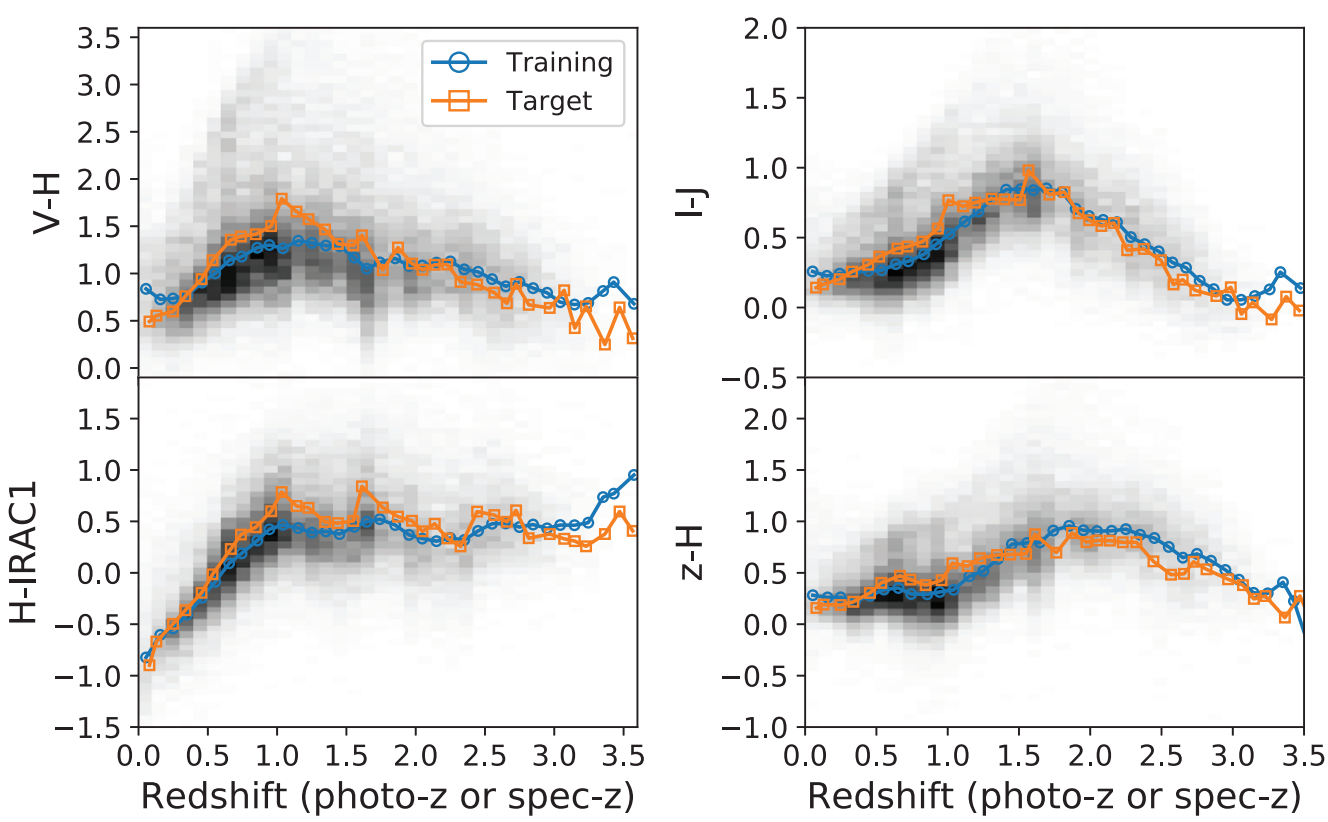

Figure 4. Observed colours in $\mathrm{AB}$ mag, $V-H, I-J, z-H, H-\mathrm{IRAC} 1$ versus redshift of the training and target sample. The greyscale is the number density of galaxies, i.e. the darker colour represents a denser region. The blue and orange points represent the median colours at each redshift bin $(\Delta z=0.1)$ for the training and target sample, respectively. The distribution of observed colours of the target sample is similar to that of the training sample. There is a clear correlation between colour and redshift with $V-H, I-J$, and $z-H$ peaking at about $z \sim 1.1,1.6$, and 1.9, respectively, when the Balmer break falls between the two bands. H-IRAC evolves strongly at $z<1$ due to the $1.6 \mu \mathrm{m}$ bump.

dust continuum whose intensity relative to the stellar population is rather poorly calibrated. Furthermore, the $3.6 \mu \mathrm{m}$ bandpass will not be available over the wide fields of Euclid and Roman. $U-B$ is also important for a secure detection of the Balmer break at low redshifts and for detecting the Lyman break at $z>2$. However, $U$ and $B$ bands in CANDELS are from ground-based telescopes (except F435W in the GOODS-S and -N fields), have shallower depth and lower resolution, so give weaker constraints on photo- $z$ estimates. We also tried to classify sources using galaxy morphological parameters such as Gini, $M_{20}$, and half-light radius, and a $H$-band magnitude. However, any combinations of morphologies and/or a magnitude, with colours, yielded worse results in deriving photometric redshifts.

Using $V-H, I-J$, and $z-H$ colours, we simply make bins in each colour with a bin size of $\Delta=0.2$ mag to build $0.2 \times 0.2 \times 0.2$ size cubes in $3 \mathrm{D}$ colour space as shown in the middle of Fig. 5. As a result, all of the galaxies in the training sample are assigned to 1286 cubes in $V-H, I-J$, and $z-H$ colour space. The bin size is chosen because it is well matched to the photometric scatter of a typical $\sim 5 \sigma$ source. In addition, using a larger bin size with less cubes results in heterogeneous templates within a cube. If on the other hand, one adopts more cubes with a smaller bin size, many cubes are not populated with templates from the training sample and the library of templates within each cube is sparsely sampled.

\subsection{Best-fitting SEDs of the training sample}

We next need to obtain the best-fitting SED of each galaxy in the training sample. This would then develop the library of templates applicable to each colour cube in three-colour space.

To obtain the best-fitting SEDs for the training set of galaxies, we fix the redshift of each galaxy to its CANDELS median photo- $z$ and fit model templates to its multiwavelength photometry. It is well known that adding zero-point offsets produce both lower scatter and outlier fraction of photometric redshifts (Dahlen et al. 2013). We therefore apply the zero-point offsets in Table A1 to the observed photometry of the training sample before doing the fits. The origin of this 'zero-point offset' is debatable since the instruments are calibrated with greater precision than the values estimated. Physically, it may be because of ignorance of galaxy templates at that level, particularly nebular emission. However, it could just as well be because of differences in how photometry is done; for instance, Skelton et al. (2014) found that on the same data, two different catalogueing techniques find differences at the 0.1-0.3 mag level. Distinguishing between these scenarios is beyond the scope of this work. In Appendix A, we 

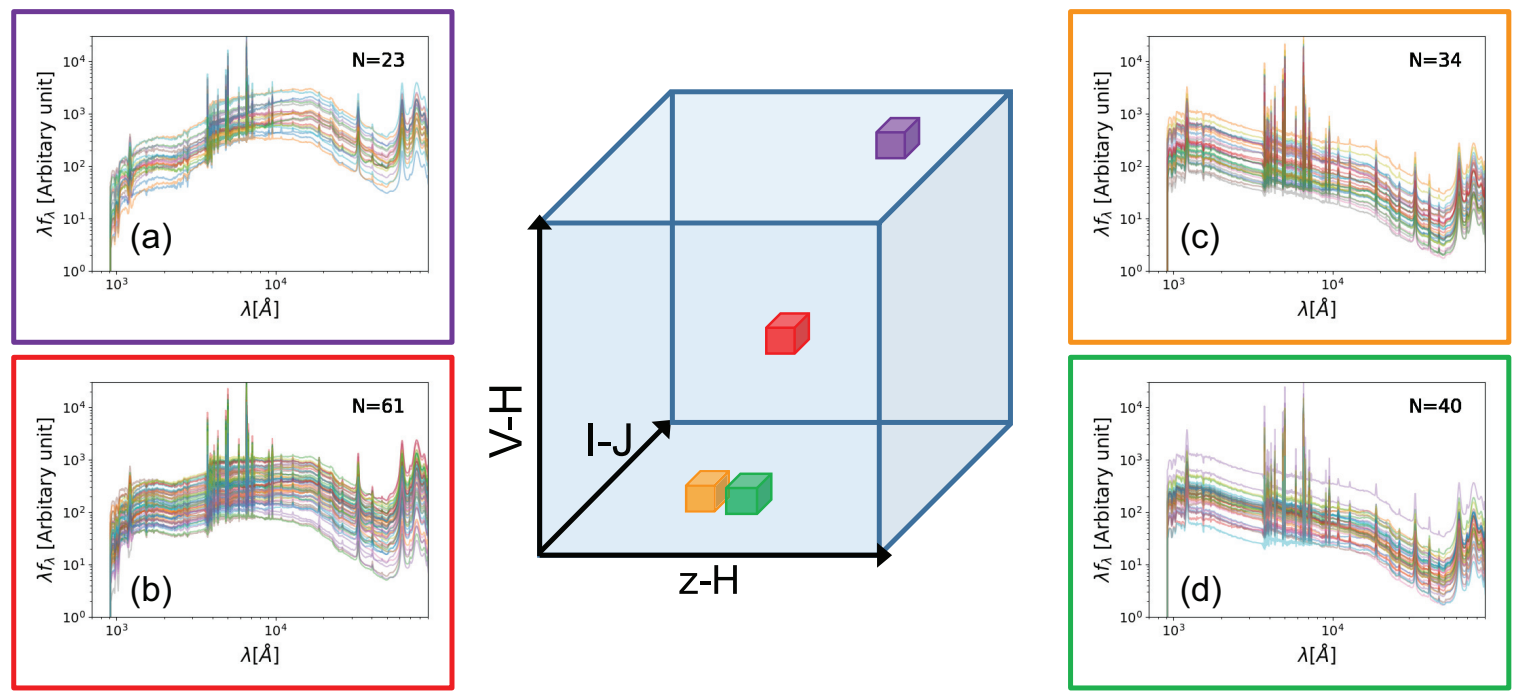

Figure 5. An illustration of the colour-cube-based template library made with the training sample in $3 \mathrm{D}$ colour space. In the middle, there are four $0.2 \times 0.2 \times 0.2$ mag size cubes at different locations in the $V-H, I-J$, and $z-H$ colour space. We also show the range of galaxy templates within the four colour cubes with the number of rest-frame SEDs in the cube; (a) for the purple cube, (b) for the red, (c) for the orange, and (d) for the green cube. For example, 23 galaxies in the training sample are assigned to the purple cube so that the purple cube-based template (a) consists of 23 rest-frame SEDs (redshifted best-fitting SEDs) of those galaxies. The red, green, and purple cubes have different types of galaxy SEDs. However, since the orange cube is located next to the green cube, their colour-cube-based templates are very similar. If a target galaxy is located in the red cube, its photometric redshift is determined using the galaxy templates (61 galaxy SEDs in b) in the red box.

compute the zero-point offsets for each individual filter and each field on the sample with spec-zs.

For the fitting, we use the EAZY (Brammer et al. 2008) code that has been widely used for large surveys, such as COSMOS (Muzzin et al. 2013), CANDELS (Dahlen et al. 2013), and 3D-HST (Skelton et al. 2014). EAZY has 12 galaxy templates derived from the flexible stellar population synthesis (FSPS) models (Conroy, Gunn \& White 2009; Conroy \& Gunn 2010) trained on the UltraVISTA photometric catalogues (Muzzin et al. 2013) with the method used for the original EAZY templates. Thus, we use the linear combination of those 12 galaxy templates derived from FSPS. We also apply the default restframe template error function scaled by a factor of 0.2 , which helps to account for systematic wavelength-dependent uncertainties in the templates (see the details from the EAZY manual ${ }^{3}$ ).

Among 39391 best-fitting SEDs of the training sample, we exclude the 4.6 per cent of fits having bad $\chi^{2}$ to avoid using poor SED fits to the data. We then shift the best-fitting SEDs to rest-frame wavelengths with CANDELS median photo-zs (hereafter, rest-frame SEDs).

\subsection{Colour-cube-based template library}

We construct the colour-cube-based template library using the restframe SEDs of the training sample. The rest-frame SED of each galaxy in the training sample is assigned to its designated cube in $V$ $-H, I-J$, and $z-H$ colour space as illustrated in Fig. 5. The colourcube-based template library is basically a collection of galaxy SEDs with similar three-colour properties. In Fig. 5, as an example, we show four sets of galaxy templates of four cubes located differently in $V-H, I-J$, and $z-H$ space. The purple, blue, and green boxes have different types of galaxy SEDs representing mostly blue starforming galaxies (green) or red passive galaxies (purple). However,

${ }^{3}$ http://www.astro.yale.edu/eazy/eazy_manual.pdf if the cubes are located closely, their SEDs are very similar like green and orange cubes. We note that there are a maximum of 1109 rest-frame SEDs in a cube.

By constraining the range of templates applicable to a galaxy using three-colour space with a photo- $z$ training sample, we are constraining the range of extinction values that may be possible among high-redshift galaxies, and constraining the range of star formation histories in each redshift bin, which helps alleviate colourredshift degeneracies.

\subsection{Photometric redshift estimates}

There are two main parts in the algorithm for determining the photometric redshift of a target sample galaxy using the colour-cubebased template library. The first is to find the correct colour cube of the target galaxy in $3 \mathrm{D}$ colour space. The second is to estimate the photo- $z$ of the target galaxy by fitting its multiwavelength photometry with the range of colour-cube-based templates with redshift as a free parameter. This is determined by minimizing the $\chi^{2}$ between the observed SED and the scaled colour-cube-based templates within that cube.

The photo- $z$ here is determined using the EAZY code modified to use the colour-cube-based template library. As before, the photometry of the target galaxy is corrected by the zero-point offsets (Table A1). We do not allow linear combination of the library of galaxy templates as is done by EAZY. For example, if three galaxies are located in the purple cube in Fig. 5, our method determines photo$z$ s of them only using the 23 galaxy templates in the purple box (plot a) and not a linear combination of subsets of the 23 templates. In other words, the rest-frame SEDs of the training sample of galaxies are assumed to be representative of, and applicable to the target sample of galaxies; the photo- $z$ of the target is thereby derived from the best-matching template. The photo- $z$ of the target galaxy is the peak of the redshift probability distribution function (i.e. $z_{\text {peak }}$ in EAZY terminology). 
Table 2. Photometric redshift results with LSST+Euclid-deep/Roman-quality photometry (ugrizYJH bands).

\begin{tabular}{lcccccccc}
\hline Field name & $z_{\text {spec }}$ range & $\mathrm{N}$ & \multicolumn{3}{c}{ This work } & \multicolumn{3}{c}{ EAZY } \\
& & & $\sigma_{\text {full }}$ & $\sigma_{\text {NMAD }}$ & $f_{\text {out }}$ & $\sigma_{\text {full }}$ & $\sigma_{\text {NMAD }}$ & $f_{\text {out }}$ \\
\hline GS & All & 1655 & 0.194 & 0.026 & 0.063 & 0.194 & 0.029 & 0.073 \\
& $z<2$ & 1410 & 0.170 & 0.025 & 0.038 & 0.164 & 0.026 & 0.045 \\
& $z>2$ & 245 & 0.269 & 0.037 & 0.208 & 0.287 & 0.047 & 0.233 \\
GN & All & 1343 & 0.157 & 0.028 & 0.056 & 0.161 & 0.028 & 0.056 \\
& $z<2$ & 1129 & 0.131 & 0.027 & 0.032 & 0.132 & 0.026 & 0.029 \\
& $z>2$ & 214 & 0.234 & 0.035 & 0.187 & 0.247 & 0.044 & 0.200 \\
\hline
\end{tabular}

One can apply the same colour ranges/combinations and the training sample to any survey. While it is possible that there could be extremely red or blue sources that are different from the training sample with CANDELS, those sources would be very rare that it would not affect the photo- $z$ precision statistics and one could use the nearest matching colour cube to provide a template library.

\section{PHOTO-Z PERFORMANCE AND VALIDATION}

We test the quality of photometric redshifts computed using the colour-cube-based template library. We compare our photo- $z$ estimates of the target sample of galaxies with their spectroscopic redshifts at $0<z<6$ and to the photometric redshifts derived using EAZY on the same sample. Although CANDELS already has photo$z$ estimates using EAZY with the original templates from Brammer et al. (2008), we re-derive photo-zs using EAZY's 12 FSPS galaxy templates with the same settings discussed in Section 3.2. We find that EAZY results in very similar or slightly better photo- $z$ estimations compared to median CANDELS photo- $z$. Since the only difference between EAZY and our analysis is that we use a more constrained template library based on our training sample, we can validate the performance of our cube-based template library by comparing to the results from EAZY.

In this section, we investigate several implementations of our colour-cube-based template library using only eight bands (LSST+Euclid-deep/Roman-like) and an inclusion of longer wavelengths, Ks $(2.2 \mu \mathrm{m})$ or IRAC $1(3.6 \mu \mathrm{m})$.

\subsection{Results with ugrizYJH photometry}

The results of our photo- $z$ fits are shown in Table 2 with statistics quantifying the accuracy of the photo- $z$ estimates. Root mean square of $\Delta_{z}$, normalized median absolute deviation (NMAD) of $\Delta_{z}$, and outlier fraction $\left(f_{\text {out }}\right)$ are defined in equation (1):

$$
\begin{aligned}
\Delta_{z} & =\left(z_{\text {spec }}-z_{\text {phot }}\right) /\left(1+z_{\text {spec }}\right), \\
\sigma_{\text {full }} & =\operatorname{rms}\left[\Delta_{z}\right], \\
\sigma_{\mathrm{NMAD}} & =1.48 \times \operatorname{median}\left[\left|\Delta_{z}\right|\right], \\
f_{\text {out }} & =N\left[\left|\Delta_{z}\right|>0.15\right] / N_{\mathrm{obj}},
\end{aligned}
$$

where the outlier fraction, $f_{\text {out }}$ is the fraction of outliers defined as objects with $\left|\Delta_{z}\right|>0.15$. Fig. 6 compares our photo- $z$ estimates to spec-zs of galaxies in the two fields with $\sigma_{\text {NMAD }}$ and $f_{\text {out }}$ listed for each field. Note that the number of target galaxies are reduced to 1655 and 1343 in GOODS-S (GS) and -N (GN), respectively, since we are not able to obtain photo-zs of 2.4 per cent galaxies in GS and 0.9 percent galaxies in GN because of two reasons. One, some of the target sample falls in cubes that do not have good templates because those cubes are only populated by SEDs with bad fits in the training sample. Two, we find that 1.6 per cent of the target sample are not located in the training cubes; about half of these are at $z_{\text {spec }}>3.6$ and comprise about 32 per cent of the $z_{\text {spec }}>3.6$ target galaxies.

The colour-cube-based template library is applicable to all redshift ranges, although the $\mathrm{S} / \mathrm{N}$ requirement in $\mathrm{U}$-band limits the training sample to $z<3.6$. We find that only three $z_{\text {spec }}>3.6$ galaxies in the target sample are excluded because they fall outside the colour range of the training sample with $\mathrm{S} / \mathrm{N}>3$ in $U$ band. If we try to derive redshifts of galaxies not located in the training cubes using the colourcube-based template library of the nearest populated colour cube, we find that it results in worse estimates. However, the CANDELS spec$\mathrm{z}$ sample has a surprising lack of high redshift galaxies with only 115 galaxies ( $\sim 2$ per cent of the spec-z sample) at $z_{\text {spec }}>3.6$ and 74 per cent of them are in the GOODS-S and $-\mathrm{N}$ fields. This is rather inadequate to investigate the photo- $z$ performance in this redshift range, and we therefore do not present the results for galaxies not located in the training cubes. In the future, we will augment the target and training samples with the results of current spectroscopic surveys, particularly at $z_{\text {spec }}>3$.

In Table 2 , we compare $\sigma_{\text {full }}, \sigma_{\mathrm{NMAD}}$, and $f_{\text {out }}$ with EAZY results. We find that our method results in smaller $\sigma_{\text {full }}, \sigma_{\mathrm{NMAD}}$, and $f_{\text {out }}$ compared to EAZY, indicating the improvement of photo- $z$ estimates. At $0<$ $z<6, \sigma_{\mathrm{NMAD}}$ of this study is $0.026(0.028)$, while EAZY yields 0.029 (0.028) in GS (GN). In Fig. 6, outliers residing below/above the red-dashed lines correspond to $\left|\Delta_{z}\right|>0.15$. We find that $f_{\text {out }}$ is about 6 per cent, similar to EAZY. $\sigma_{\text {full }}$ is 0.194 and 0.157 in GS and GN. We improve the photo- $z$ precision by about 10 per cent and the outlier fraction is decreased by about 13 per cent compared to EAZY in GS, while in GN $f_{\text {out }}$ and $\sigma_{\text {NMAD }}$ are almost identical between the two techniques. However, we find that at $z>2$, our method shows a significant improvement on the photo- $z$ estimates in both GS and GN as shown in Table 2. At $z<2$, our method results in smaller statistics compared to EAZY with galaxies in GS, while $\sigma_{\mathrm{NMAD}}$ and $f_{\text {out }}$ in GN are slightly larger than ones from EAZY. Overall, we obtain better photo- $z$ performance with GS compared to GN. This might be due to the fact that $U$ band in GS is slightly deeper than GN. The $5 \sigma$ limiting depth of $U$-band VLT/VIMOS in GS is $27.97 \mathrm{AB}$ mag, while $\mathrm{GN} U$-band KPNO/Mosaic has $5 \sigma$ of $26.7 \mathrm{AB}$ mag. With the colour-constrained templates limiting the range of extinctions, the deeper $U$ band of GS can classify high- and low-redshift galaxies better in the fitting.

We also plot $\sigma_{\text {full }}, \sigma_{\mathrm{NMAD}}$, and $f_{\text {out }}$ as a function of redshift in Fig. 7 . At all redshifts considered here, photo- $z$ precision is relatively high with $0.02<\sigma_{\mathrm{NMAD}}<0.04$. In particular, at $z>2, \sigma_{\mathrm{NMAD}}$ of our method is significantly small, $\sigma_{\mathrm{NMAD}} \sim 0.03-0.04$, comparing to EAZY with $\sim 0.06$ at $2<z<3$, indicating that we can improve the photo- $z$ precision by about 30 per cent using the colour-cube-based 

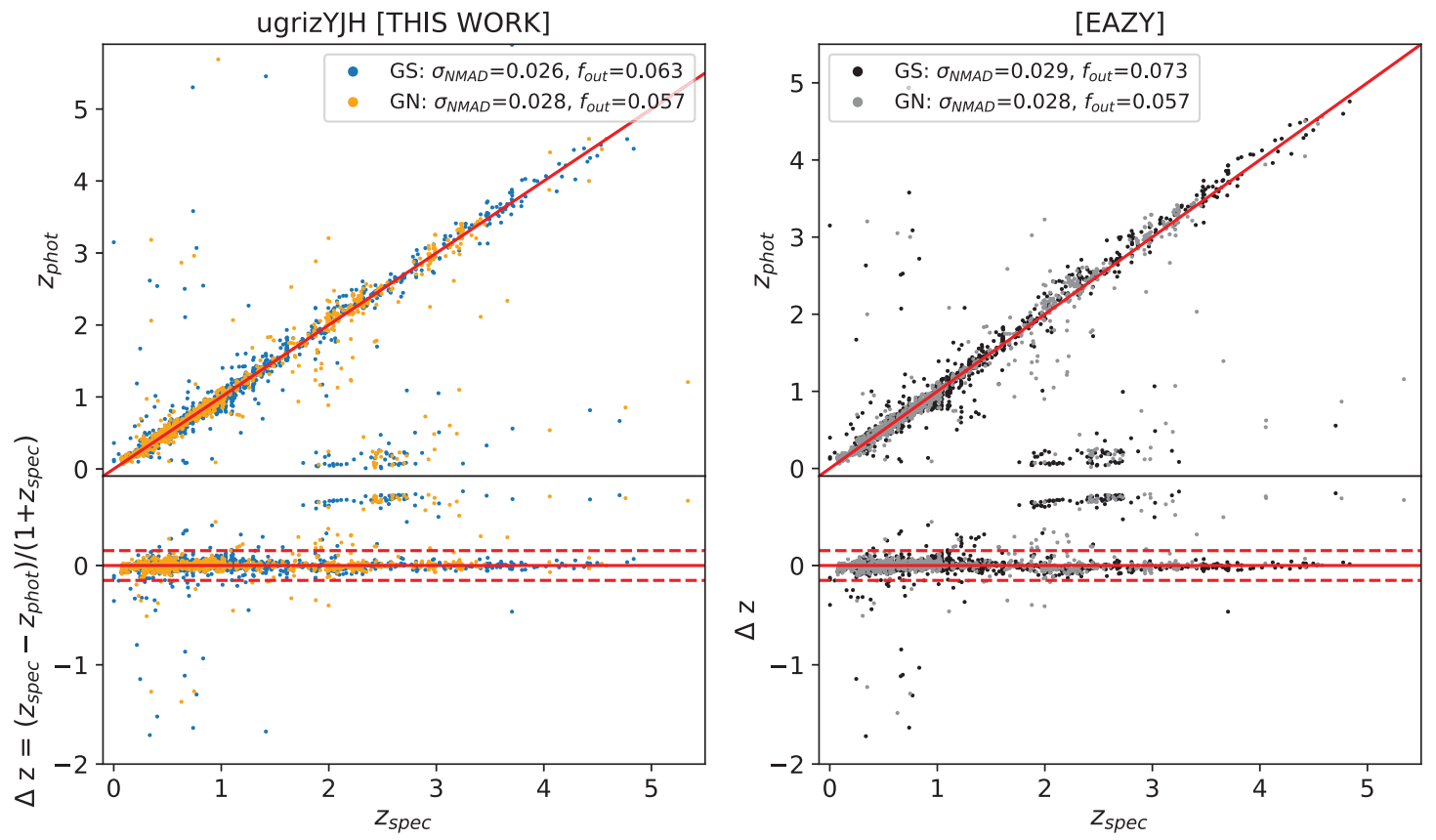

Figure 6. Comparison between spectroscopic redshifts $\left(z_{\text {spec }}\right)$ and photo- $z$ estimates $\left(z_{\text {phot }}\right)$ of the target sample in GOODS-S (GS) and GOODS-N (GN). Left: A result from our method with GOODS-S (blue) and GOODS-N (orange) galaxies. Right: The result from EAZY for the same galaxies (GS: black, GN: grey). We plot $z_{\text {phot }}$ versus $z_{\text {spec }}$ in the top panel and $\Delta_{z}\left(=\left(z_{\text {spec }}-z_{\text {phot }}\right) /\left(1+z_{\text {spec }}\right)\right)$ versus $z_{\text {spec }}$ in the bottom panel with $\sigma_{\text {NMAD }}$ and the outlier fraction $f_{\text {out }}$ measured for each field. $\Delta_{z}=0$ is shown as a red line with dashed lines indicating $\left|\Delta_{z}\right|=0.15$. Galaxies located below/above the dashed lines are classified as outliers. Here, we use LSST+Euclid-deep/Roman-quality multiband photometry to derive photo- $z$ using the colour-cube-based template library made from $V-H, I-$ $J$, and $z-H$ colours. Our method results in an improved photo-z precision of $\sigma_{\mathrm{NMAD}}=0.26-0.28$ and smaller outlier fraction of about 6 per cent comparing to EAZY with $\sigma_{\mathrm{NMAD}}=0.028-0.029$ and $f_{\text {out }}=6-7$ per cent.

template library at $z>2$. Other statistics, $\sigma_{\text {full }}$ and $f_{\text {out }}$ are smaller than ones from EAZY at $1.5<z<3.5$. They are, however, similar to EAZY at lower redshifts and the highest redshift bin $(z>3.5)$. Our result shows that the colour-cube-based template library is powerful for estimating photometric redshifts when the number of photometric bands is limited, especially at high redshifts.

\subsection{Results with an inclusion of IRAC 1 or Ks bands}

It has been shown that the derivation of photometric redshifts benefit from having multiple narrow bands and having broad wavelength coverage (Dahlen et al. 2010), especially if they straddle strong features in galaxy SEDs such as the Balmer break and $1.6 \mu \mathrm{m}$ bump. It is therefore worth assessing if the addition of longer wavelength bands to the planned ugrizYJH photometry would be beneficial for photo- $z$ derivation. We investigate here the redshift accuracy when we add longer near-infrared bands, Ks $(2.2 \mu \mathrm{m})$ or IRAC $1(3.6 \mu \mathrm{m})$ to the ugrizYJH bands.

We first derive photo-zs using our method by including the IRAC 1 band, i.e. nine bands adding IRAC 1 to the ugrizYJH bands. The results are shown in Table 3. In comparison to the result only using ugriz YJH (Table 2), we are able to improve the overall performance of photo-zs when the IRAC 1 band is included. We obtain a smaller $\sigma_{\text {NMAD }}=0.025$ in both GS and GN. The fraction of outliers decreases from 6.3 per cent to 5.1 per cent for the GS and 5.6-5.2 per cent for the GN. Likewise, we find $\sigma_{\text {full }}$ drops dramatically in both fields, to 0.141 (GS) and $0.130(\mathrm{GN})$, which corresponds to about a 27 per cent and 17 per cent decrease, respectively. This implies that the inclusion of the IRAC 1 band has a significant impact on reducing outliers. This is particularly relevant for the $40 \mathrm{deg}^{2}$ Euclid-deep fields, which are the target of Spitzer observations achieving depths of $5 \sigma=24.6$
AB mag (PI: P. Capak), although this depth is shallower than the IRAC data used here.

Next, we derive photo-zs with nine bands adding Ks band to the ugrizYJH. Ks band photometry in GS is quite deep with VLT/HAWK-I ( $5 \sigma$ depth of $26.45 \mathrm{AB}$ ), while the GN Ks-band is shallower, $24.4 \mathrm{AB}$ mag. As shown in Table 3, the inclusion of the shallow $K s$-band photometry has only a marginal effect for galaxies in GN when compared to the result without $K s$ band. The relatively low impact on the photometric redshifts when including $K s$ band could be attributed to the fact that the $H$ band from HST already provides a tight constraint on the SED longwards of the Balmer break and the $1.6 \mu \mathrm{m}$ bump has a relatively small effect on photo- $z$ estimation at the redshift range of interest. Despite having deeper $K s$ band in GS, we obtain worse results with a photo- $z$ precision of $\sigma_{\mathrm{NMAD}}=0.29$. The origin of this discrepancy is a puzzle and might require a careful re-evaluation of the HAWK-I photometry.

\section{CONCLUSIONS: AN INTRODUCTION OF NEW TEMPLATE LIBRARIES}

We investigate a new method for improving the accuracy of photo- $z$ estimates for future wide-area surveys that will be deep and have limited multiwavelength coverage. There are two unique features in our method. To have a large unbiased galaxy sample, we select the training sample of galaxies from CANDELS to have photometric redshifts that are a median of 11 different photo- $z$ codes. Although the survey area is small (about $0.25 \mathrm{deg}^{2}$ ) compared to future widearea surveys, CANDELS is the only survey having de-blended photometry including deep near-IR bands and having spatial resolution comparable to Roman and Euclid. We then populate 3D colour space, $V-H, I-J$, and $z-H$ with the best-fitting SEDs for this training 

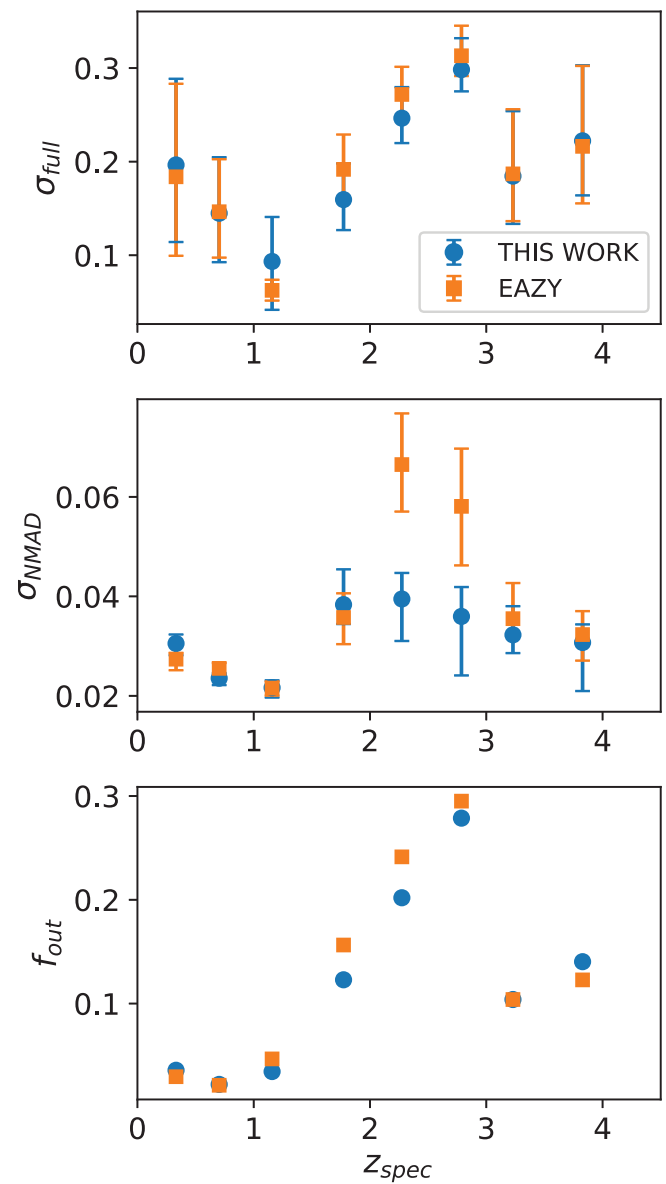

Figure 7. Three photo- $z$ statistics, $\sigma_{\text {full }}, \sigma_{\mathrm{NMAD}}$, and $f_{\text {out }}$ as a function of spec-z $\left(z_{\text {spec }}\right)$. Bin size is $\Delta z_{\text {spec }}=0.5$, while the last bin include galaxies at $3.5<z_{\text {spec }}<5.5$. Statistics measured in each redshift bin are shown on the $Y$-axis for this work (blue) and EAZY (orange). $X$-axis is the median $z_{\text {spec }}$ in each bin. The number of galaxies in each redshift bin is $644,1223,493$, $179,203,122,77,57$, respectively. The error bars of $\sigma_{\text {full }}$ and $\sigma_{\mathrm{NMAD}}$ are determined by bootstrapping the sample. Our photo- $z$ performance at $1.5<$ $z<3.5$ is better with smaller values of $\sigma_{\text {full }}, \sigma_{\mathrm{NMAD}}$, and $f_{\text {out }}$ than ones from EAZY, while similar to EAZY at $z<1.5$ and $z>3.5$. In particular, our method has a very high precision with $\sigma_{\mathrm{NMAD}}<0.04$ at $z>2$ compared to EAZY with $<0.06$, indicating about 30 per cent improved performance at $z>2$.

Table 3. Derived Photo- $z$ precision when including IRAC1 or Ks bands.

\begin{tabular}{lcccc}
\hline & Field name & $\sigma_{\text {full }}$ & $\sigma_{\text {NMAD }}$ & $f_{\text {out }}$ \\
\hline With IRAC 1 & GS & 0.141 & 0.025 & 0.051 \\
& GN & 0.130 & 0.025 & 0.052 \\
With $K$ band & GS & 0.168 & 0.029 & 0.063 \\
& GN & 0.158 & 0.027 & 0.060 \\
\hline
\end{tabular}

sample to derive a colour-cube-based template library trained with high-quality multiwavelength CANDELS photometry.

We apply our colour-cube-based template library to a target sample of galaxies with ugrizYJH photometry (LSST+Euclid/Roman-like) and derive their photo-zs. We quantify the performance of our method for determining photo-zs by comparing with spec-zs. We derive a photo- $z$ precision of $\sigma_{\mathrm{NMAD}}=0.026-0.028$ and outlier fraction of about 6 percent for galaxies in the GOODS-S and $-\mathrm{N}$ fields. We compare our results with EAZY using their templates and find that our method improves the photo- $z$ precision by about 10 per cent and reduces the outliers by about 13 percent. In particular, the colourcube-based template library is very effective at $2<z<3$ with significantly small $\sigma_{\mathrm{NMAD}}$ of $\sim 0.04$, compared to EAZY that yields $\sigma_{\text {NMAD }} \sim 0.06$. We also investigate the inclusion of additional longer wavelength photometry, e.g. Ks band or IRAC1 $(3.6 \mu \mathrm{m})$, to the ugrizYJH photometry. Including deep IRAC1 photometry results in smaller $\sigma_{\mathrm{NMAD}}=0.025$ and has the most significant impact on reducing outliers. However, the inclusion of $K s$ band has marginal to no effect on the photometric redshifts mainly due to the fact that the deeper $H$-band data from $H S T$ already provides a tight constraint on the galaxy SEDs.

The main advantage of having a photo- $z$ training sample is that it allows the SEDs of dusty galaxies to be constrained and distinguished from high- $z$ Lyman break galaxies. Having purely a spec-z training sample would make it challenging to constrain the SEDs of such galaxies since spec-z samples tend be biased against dusty galaxies. This allows for better redshift determination of low-redshift dusty galaxies, which would be otherwise erroneously fitted to be highredshift galaxies. Furthermore, having such a photo- $z$ training sample constrains the range of extinction values that may be possible among high-redshift galaxies and constrains the range of star formation histories in each redshift bin. Both these effects cause shifts in the best estimate photometric redshifts that is reducing the scatter at high redshift. While it is possible that there could be sources with SEDs that are radically different from the training sample as one expands to wider areas, those sources will likely fail but would be rare enough that it would not affect the photo- $z$ statistics.

Overall, our result clearly shows that the colour-cube-based template library trained with CANDELS data in multidimensional colour space is a powerful tool for determining photometric redshifts using the template-fitting approach, particularly for future large extragalactic surveys that will provide deep ugrizYJH photometry. These results will be highly applicable to the LSST and Euclid-deep fields which span $40 \mathrm{deg}^{2}$ and span similar depths as those sampled in this study. They can also be applied to the LSST+Roman high latitude survey sky area that will span $2200 \mathrm{deg}^{2}$.

\section{ACKNOWLEDGEMENTS}

This work is funded by NASA/Euclid grant 1484822 and Joint Survey Processing (JSP) effort at Infrared Processing and Analysis Centre (IPAC), which has been provided by NASA grant 80NM0018F0803. The data used in this work are based on observations taken by the CANDELS MultiCycle Treasury Program with the NASA/ESA HST, which is operated by the Association of Universities for Research in Astronomy, Inc., under NASA contract NAS5-26555. The authors thank Andreas Faisst for thoughtful comments that improved this manuscript and Gabe Brammer for helpful ideas that improved our analysis. We also thank the anonymous referee for very useful comments that helped to improve the presentation of the paper.

\section{DATA AVAILABILITY}

The CANDELS multiwavelength catalogues and photometric redshifts catalogues used in this study are publicly available on the Mikulski Archive for Space Telescopes: https://archive.stsci.edu/pr epds/candels/. The photometric redshift estimations underlying this article will be shared on reasonable request to the corresponding author. 


\section{REFERENCES}

Barro G. et al., 2019, ApJS, 243, 22

Bilicki M. et al., 2018, A\&A, 616, 69

Brammer B. B., van Dokkum P. G., Coppi P., 2008, ApJ, 686, 1503

Carrsco Kind M., Brunner R. J., 2013, MNRAS, 432, 1483

Carrsco Kind M., Brunner R. J., 2014, MNRAS, 438, 3409

Collster A. A., Lahav O., 2004, PASP, 116, 345

Conroy C., Gunn J. E., 2010, ApJ, 712, 833

Conroy C., Gunn J. E., White M., 2009, ApJ, 699, 486

Dahlen T. et al., 2010, ApJ, 724, 425

Dahlen T. et al., 2013, ApJ, 775, 93

Fotopoulou S., Paltani S., 2018, A\&A, 619, 14

Galametz A. et al., 2013, ApJS, 206, 10

Grogin N. A. et al., 2011, ApJS, 197, 35

Guo Y. et al., 2013, ApJS, 207, 24

Hasinger G. et al., 2018, ApJ, 858, 77

Hoyle B., Rau M. M., Zitlau R., Seitz S., Weller J., 2015, MNRAS, 449, 1275

Hyole B., 2017, Astron. Comput., 16, 34

Ilbert O. et al., 2006, A\&A, 457, 841

Ilbert O. et al., 2009, ApJ, 690, 1236

Joint Survey Processing Final Report et al., 2019, BAAS, 51, 44

Koekemoer A. M. et al., 2011, ApJS, 197, 36

Laigle C. et al., 2019, MNRAS, 486, 5104

Laureijs R. et al., 2011, preprint (arXiv:1110.3193)

Lilly S. J. et al., 2007, ApJS, 172, 70

Masters D. C. et al., 2015, ApJ, 813, 53

Masters D. C. et al., 2017, ApJ, 841, 2

Masters D. C. et al., 2019, ApJ, 877, 81

Merlin E. et al., 2016, A\&A, 595, 97

Muzzin A. et al., 2013, ApJS, 206, 8

Nayyeri H. et al., 2017, ApJS, 228, 7

Rau M. M. et al., 2015, MNRAS, 452, 3710

Salvato M. et al., 2009, ApJ, 690, 1250

Salvato M., Ilbert O., Hoyle B., 2019, Nat. Astron., 3, 212

Sánchez C. et al., 2014, MNRAS, 445, 1482

Sawicki M., 2002, ApJ, 124, 3050

Schlafly E. F., Finkbeiner D. P., 2011, ApJ, 737, 103

Skelton R. E. et al., 2014, ApJS, 214, 24

Speagle J. S., Eisenstein D. J., 2017a, MNRAS, 469, 1186
Speagle J. S., Eisenstein D. J., 2017b, MNRAS, 469, 1205

Spergel D. et al., 2015, preprint (arXiv:1503.03757)

Stefanon M. et al., 2017, ApJS, 229, 32

Tasca L. A. M. et al., 2017, A\&A, 600, 110

van der Wel A. et al., 2014, ApJ, 788, 28

van der Wel A. et al., 2016, ApJS, 223, 29

\section{APPENDIX A: ZERO-POINT OFFSETS}

As described in Ilbert et al. (2006), Dahlen et al. (2013), and Skelton et al. (2014), the application of offsets to the photometry can improve photometric redshift fits. These are called zero-point offsets but in general, since the instrument photometry is calibrated with high accuracy, is unlikely to be due to actual errors in zero-points. It is therefore thought that any offsets are likely due to the mismatch between the SED templates and real galaxy SEDs (Guo et al. 2013), although inconsistent isophotal apertures across bands may also be a contributing factor. Zero-point offsets are determined for individual filters and vary in different fields. We measure the zero-point offset of each band by fitting the EAZY FSPS templates to the observed photometry of galaxies with spectroscopic redshifts as in Skelton et al. (2014). The offsets are computed iteratively by minimizing the median differences between the measured photometry and bestfitting templates integrated over the bandpasses in a particular field. This correction is then applied to the photometry of all sources. In principle, the correction should be calculated on a spec-z sample that is distinct from the sample on which the quality of photo$z$ is measured. However, due to the limited number of spec-zs available, these corrections are applied on the same sample. With future spectroscopic surveys, this is likely to be improved.

The listed zero-point offset corrections in Table A1 have been applied to the photometry when we obtain the best-fitting SED of each galaxy in the training sample in Section 3.2 as well as when we derive the photometric redshifts of the target sample using the colour-cube-based template library in Section 3.4. The values in the table are multiplicative numbers to be applied to the flux densities in the corresponding filters.

Table A1. Zero-point offsets for individual filters in five fields.

\begin{tabular}{|c|c|c|c|c|c|c|c|c|c|}
\hline \multicolumn{2}{|c|}{ GOODS-S } & \multicolumn{2}{|c|}{ GOODS-N } & \multicolumn{2}{|l|}{ UDS } & \multicolumn{2}{|l|}{ EGS } & \multicolumn{2}{|c|}{ COSMOS } \\
\hline Filter & zp offset & Filter & zp offset & Filter & zp offset & Filter & zp offset & Filter & zp offset \\
\hline U Blanco & 1.0404 & U KPNO & 0.9483 & U CFHT & 1.1115 & u CFHT & 0.9980 & u CFHT & 0.9070 \\
\hline U VLT & 1.0304 & F435W & 1.0616 & B Subaru & 0.9999 & g & 0.9619 & g & 0.9114 \\
\hline F435W & 0.9855 & F606W & 0.9928 & V & 0.9902 & $\mathrm{r}$ & 0.9960 & $\mathrm{r}$ & 0.9506 \\
\hline F606W & 0.9929 & F775W & 0.9811 & $\mathrm{R}$ & 0.9144 & $\mathrm{i}$ & 0.9786 & $\mathrm{i}$ & 0.9415 \\
\hline F775W & 0.9892 & F814W & 0.9826 & $\mathrm{i}$ & 0.9878 & $\mathrm{Z}$ & 0.9763 & $\mathrm{z}$ & 0.9503 \\
\hline F814W & 0.9910 & F850LP & 0.9531 & $\mathrm{z}$ & 0.9960 & F606W & 0.9315 & B Subaru & 0.9888 \\
\hline F850LP & 0.9674 & F105W & 0.9900 & F606W & 1.0518 & F814W & 0.9624 & F606W & 0.9476 \\
\hline F098M & 1.0210 & F125W & 1.0020 & F814W & 0.9834 & F125W & 1.0025 & F814W & 0.9784 \\
\hline F105W & 1.0053 & F140W & 0.9915 & Y HAWK-I & 1.0144 & F140W & 0.9907 & F125W & 1.0183 \\
\hline F125W & 1.0070 & F160W & 1.0000 & F125W & 1.0065 & F160W & 1.0000 & F160W & 1.0000 \\
\hline F160W & 1.0000 & Ks Subaru & 0.9540 & F160W & 1.0000 & J1 NEWFIRM & 0.9255 & Y UVISTA & 0.9012 \\
\hline Ks VLT/ISAAC & 1.0675 & Ks CFHT & 0.9373 & Ks VLT/HAWK-I & 1.0016 & $\mathrm{~J} 2$ & 0.9117 & Ks UVIST & 0.9202 \\
\hline Ks VLT/HAWK-I & 1.0100 & IRAC 1 & 1.0070 & K UKIRT/WFCAM & 1.0039 & $\mathrm{~J} 3$ & 0.9248 & J1 NEWFIRM & 0.8942 \\
\hline IRAC 1 & 0.9616 & IRAC 2 & 0.9871 & IRAC 1 & 1.0006 & H1 & 0.9060 & $\mathrm{~J} 2$ & 0.9034 \\
\hline IRAC 2 & 1.0175 & IRAC 3 & 1.0404 & IRAC 2 & 0.9825 & $\mathrm{H} 2$ & 0.9316 & $\mathrm{~J} 3$ & 0.9223 \\
\hline IRAC 3 & 0.9940 & IRAC 4 & 1.0507 & IRAC 3 & 0.9447 & Ks WIRCAM & 0.9636 & H1 & 0.8969 \\
\hline IRAC 4 & 1.0116 & & & IRAC 4 & 0.9848 & IRAC 1 & 0.9857 & $\mathrm{H} 2$ & 0.8777 \\
\hline & & & & & & IRAC 2 & 0.9783 & IRAC 1 & 0.9372 \\
\hline & & & & & & IRAC 3 & 1.0594 & IRAC 2 & 0.9166 \\
\hline & & & & & & IRAC 4 & 0.7614 & IRAC 3 & - \\
\hline
\end{tabular}


Table A1 - continued

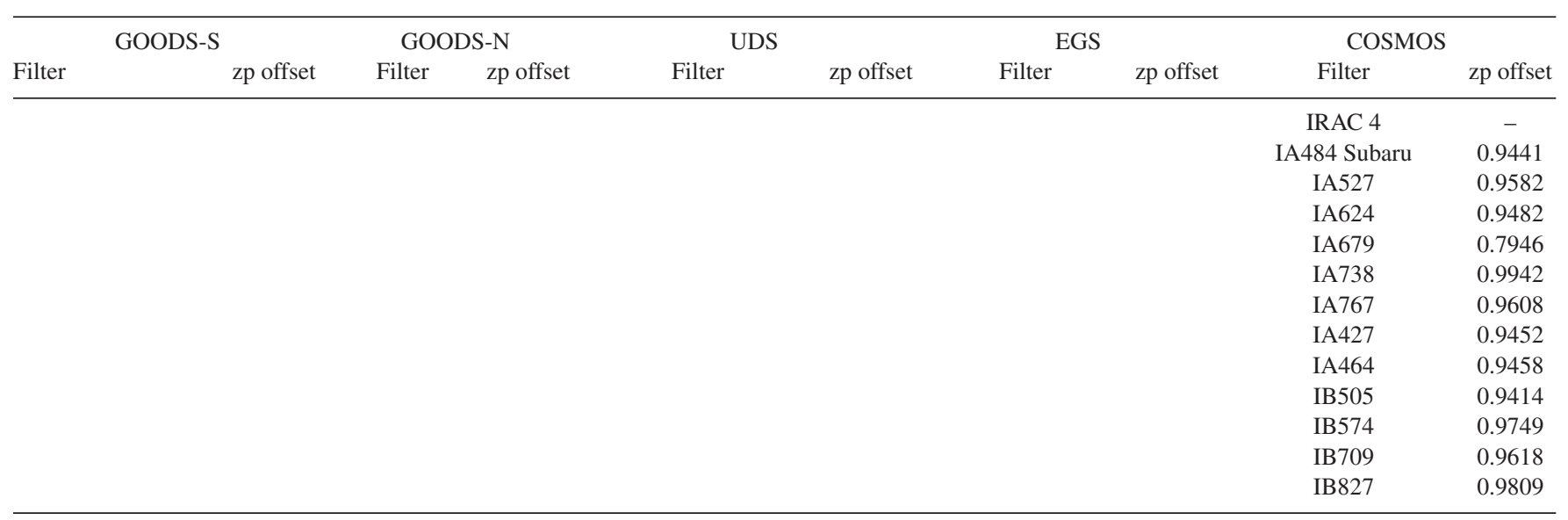

This paper has been typeset from a $\mathrm{T}_{\mathrm{E}} \mathrm{X} / \mathrm{LT} \mathrm{E} \mathrm{X}$ file prepared by the author. 